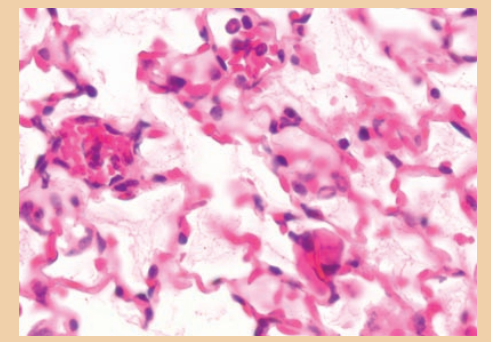

Transfusion-related acute lung injury (TRALI) is a major problem in transfusion medicine and has emerged as the leading cause of death from a blood transfusion. There is a lack of mechanistic insight into the pathogenesis of transfusion-related acute lung injury, primarily due to the absence of an in vivo model. Looney and colleagues now describe a new in vivo mouse model of transfusion-related acute lung injury (pages 1615-1623). Transfusion of an MHC I mAb into BALB/c mice produced acute lung injury with increased excess lung water, increased lung vascular and lung epithelial permeability, and decreased alveolar fluid clearance. Half of the mice died 2 hours after antibody administration. Within those 2 hours, neutrophil sequestration in the lung microvasculature occurred concomitantly with acute peripheral blood neutropenia. Depletion of neutrophils protected mice from the lung injury. While $F c R \gamma^{-/-}$mice were resistant to lung injury, adoptive transfer of wild-type neutrophils into the $F c R \gamma^{-/-}$animals restored susceptibility to TRALI. With their clinically relevant in vivo mouse model of TRALI, the authors therefore suggest that the mechanism of lung injury is dependent on neutrophils and their Fcy receptors.

\section{New insights into bipolar disorder}

Calcium is a ubiquitous intracellular signaling molecule required for initiating and regulating a wide range of neuronal functions, including neurotransmitter release, synaptic plasticity, neurite outgrowth, and neurodegeneration. Now Schlecker and colleagues investigate the interaction between the principal intracellular calcium release channel in cells, the inositol 1,4,5-trisphosphate receptor (InsP $\left.{ }_{3} \mathrm{R}\right)$, and the calcium-binding protein neuronal calcium sensor-1 (NCS-1) (pages 1668-1674). NCS-1 was found to modulate the activity of the $\mathrm{Ins}_{3} \mathrm{R}$. Interaction between the 2 proteins was observed with purified proteins and in intact cells. NCS-1 dramatically increased the opening rate of the $\mathrm{InsP}_{3}$-gated channel in both calciumdependent and -independent manners. As NCS-1 has been found to be elevated in the cortex of bipolar patients, the authors show that levels of lithium used for treating bipolar patients attenuate the enhanced signaling when NCS-1 is overexpressed. These data suggest a new mechanism of action for lithium and suggest that the interaction of Ins $\mathrm{P}_{3} \mathrm{R} 1$ and NCS- 1 is an essential component of the pathobiology of bipolar disorder.

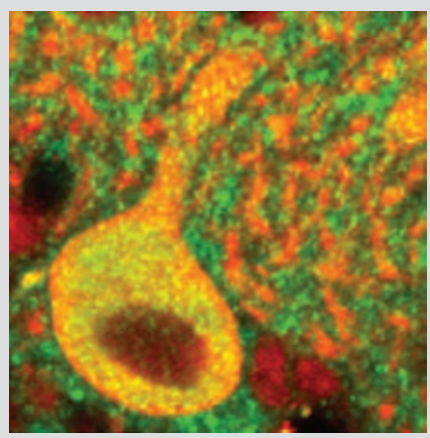

\section{A noninvasive method for measuring $\beta$ cell mass}

Diabetes results from a reduction in pancreatic $\beta$ cell mass (BCM) leading to insufficient insulin secretion and hyperglycemia. Measurement of insulin secretion is currently used as a surrogate measure of BCM; however, serum insulin concentrations provide an imprecise index of BCM. Now Souza and colleagues describe a reliable noninvasive measure of BCM (pages 1506-1513). The authors exploit the find-

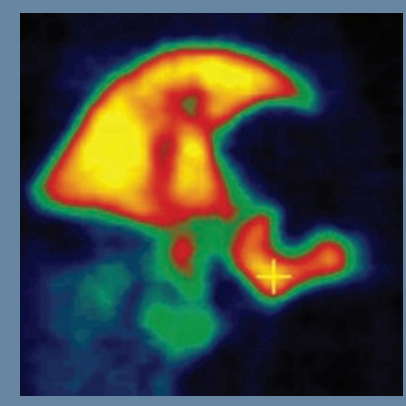
ing that type 2 vesicular monoamine transporters (VMAT2) are expressed in human islet $\beta$ cells, as well as in tissues of the CNS. Because the radioligand $\left[{ }^{11} \mathrm{C}\right]$ dihydrotetrabenazine $\left(\left[{ }^{11} \mathrm{C}\right] \mathrm{DTBZ}\right)$ binds specifically to VMAT2 and is currently used in clinical imaging of the brain, the authors were able to use $\left[{ }^{11} \mathrm{C}\right] \mathrm{DTBZ}$ to estimate BCM in a rat model of spontaneous type 1 diabetes. In longitudinal PET studies, the authors saw a significant decline in pancreatic uptake of $\left[{ }^{11} \mathrm{C}\right] \mathrm{DTBZ}$ that preceded the loss of glycemic control in the diabetic rat. These studies suggest that PET-based quantitation of VMAT2 receptors could provide a noninvasive measurement of BCM that could be used to study pathogenesis of diabetes and to monitor therapeutic interventions. 